\title{
Transform-domain Solutions of Poisson's Equation with Applications to the Asymptotic Variance
}

\author{
Koen De Turck, Sofian De Clercq, Sabine Wittevrongel, \\ Herwig Bruneel, and Dieter Fiems \\ Department of Telecommunications and Information Processing, \\ Ghent University \\ Sint-Pietersnieuwstraat 41, B-9000 Gent, Belgium \\ Email: \{kdeturck, sdclercq, sw, hb, df $\} @$ telin.UGent.be
}

\begin{abstract}
Recent years have seen a considerable increase of attention devoted to Poisson's equation for Markov chains, which now has attained a central place in Markov chain theory, due to the extensive list of areas where Poisson's equation pops up: perturbation analysis, Markov decision processes, limit theorems of Markov chains, etc. all find natural expression when viewed from the vantage point of Poisson's equation. We describe how the use of generating functions helps solve Poisson's equation for different types of structured Markov chains and for driving functions, and point out some applications. In particular, we solve Poisson's equation in the transform domain for skip-free Markov chains and Markov chains with linear displacement. Closed-form solutions are obtained for a class of driving functions encompassing polynomial functions and functions with finite support.
\end{abstract}

\section{Introduction}

In this paper, we investigate the use of transform-domain techniques to solve Poisson's equations associated with various classes of Markov chains. Poisson's equation (PE) has in recent years been acknowledged to be one of the central concepts of Markov chains, almost equal in importance to the steady-state equation. In order to advance the exposition, we introduce some notation. Let $(\Omega, \mathcal{F}, \mathbb{P})$ be a probability space and let $\left\{X_{n}\right\}, n \in \mathbb{Z}$, be a time-homogeneous Markov chain, taking values in a countable state space $\mathcal{X}$ (this is not a strict necessity, but as our example Markov chains will all have a countable state space, we have opted to avoid the technicalities of dealing with more general state spaces). The transition probabilities $p_{i j}=\mathbb{P}\left[X_{n+1}=j \mid X_{n}=i\right], i, j \in \mathcal{X}$ are recorded in a (possibly countably infinite) transition matrix $P$.

Two linear systems of equations are commonly associated with such a Markov chain. The first is the invariance equation that says that the stationary distribution recorded in row vector $\boldsymbol{\pi}=\left[\pi_{i}\right]_{i \in \mathcal{X}}$, provided it exists, satisfies:

$$
\pi_{j}=\sum_{i \in \mathcal{X}} \pi_{i} p_{i j} \quad \text { or in matrix notation: } \boldsymbol{\pi}=\boldsymbol{\pi} P .
$$


The importance of this equation is such, that the expression 'solving a Markov chain' is colloquially taken to mean almost unambiguously solving equation 1 .

The second equation, Poisson's equation (PE) - perhaps somewhat surprisingly - has only quite recently been put on a comparable level of importance. Its formulation requires a function on the state space $f: \mathcal{X} \rightarrow \mathbb{R}$, which is depending on the application referred to as the driving function, cost function or reward function. Given this function $f$, we aim to assign a value $h_{i}$ to each state $i$ of the chain, which records the relative long-term cost of starting from state $i$ as compared to others. Indeed, Poisson's equation is equal to:

$$
h_{i}=f_{i}-\sum_{j \in \mathcal{X}} \pi_{j} f_{j}+\sum_{j \in \mathcal{X}} p_{i j} h_{j} .
$$

In words, the relative value of state $i$ is equal to the cost of state $i$, minus the average cost in stationarity, plus the expected relative value of the Markov chain one step in the future. Readers may notice the similarity with Bellman's equation [3] or with dynamic programming equations in general. Note that the term Poisson's equation is not native to probability theory, but hails from the theory of partial differential equations and was chosen due to certain similarities between the two. A slight rearrangement of terms results in the following perhaps more familiar form:

$$
(I-P) \mathbf{h}=(I-\mathbf{1} \boldsymbol{\pi}) \mathbf{f},
$$

where $I$ denotes the identity matrix of appropriate dimension, $\mathbf{1}$ is a column vector with element 1 on each row and $\mathbf{f}$ and $\mathbf{h}$ are the value and cost column vectors respectively.

Solutions for the PE typically proceed from the fundamental kernel or deviation matrix which will be introduced in the Sec. 2 .

A variant equation that has shown to be relevant for applications is the discounted Poisson's equation:

$$
h_{i}-\gamma \sum_{j} p_{i j} h_{j}=f_{i},
$$

or in matrix notation:

$$
(I-\gamma P) \mathbf{h}=\mathbf{f} .
$$

where $\gamma$ is referred to as the discount factor. The idea behind this equation is that costs further in the future should have a smaller impact on the value than short-term costs. In this paper, we will concentrate on the original Poisson equation.

Neveu [9] seems to have been the first to coin the term Poisson's equation in a Markov context. Over the years, many new results were discovered, both from a practitioner's point of view, as more applications were discovered, such as perturbation [5], limit theorems [10], constructing approximative models [7], as well as from a theoretical point of view, which has resulted in a better, and perhaps almost complete understanding as to when solutions of PE exist. A key concept in this regard is $V$-uniform ergodicity [6], which provides sufficient 
conditions that seem close to necessary. Its importance was first noticed by Hordijk and Spieksma [14], and taken to full generality by Meyn and Tweedie [6].

It is to be expected that the importance of Poisson's equation will further increase, as control of queueing systems gets a more pronounced place next to mere performance analysis. In this paper, we tackle the Poisson's equation with transform-domain tools, which are since long popular in applied probability and in queueing theory in particular [1,2]. Related work includes the results of Koole [13], who derived closed-form expressions for the deviation matrix of birth-and-death-processes. Related work also includes $[17,18]$, which uses the matrix-analytic paradigm but, in contrast to this paper, is restricted to finite state spaces.

The outline of the rest of this paper is as follows. In Sec. 2 we outline the general recipe and work out in detail a solution for the PE for some often encountered types of Markov chains, and in Sec. 3, we show an application to the computation of the asymptotic variance. Finally, we shed a light on further applications and offer some concluding remarks in Sec. 4.

\section{Main Results}

In this section, we outline a general recipe for solving Poisson's equation with transform-domain techniques. We will in the current paper generally take the stance that 'the spirit is more important than the letter', and will only briefly address such technical issues as the convergence, existence and uniqueness of solutions for the occurring expressions. As tools for proving such existence exist [6], a rigourous application of the results of this paper thus consists of proving the existence of a PE solution with such means, calculating the solution with the computational method of this paper, and verifying that it indeed satisfies PE afterwards.

Let us assume for a moment that $\mathcal{X}=\mathbb{N}$ and introduce some notation. For a function on the state space recorded in a (either row or column) vector $\mathbf{v}=\left[v_{i}\right]_{, i \in \mathbb{N}}$, let $\mathcal{G}_{\mathbf{v}}$ denote the corresponding generating function, i.e.

$$
\mathcal{G}_{\mathbf{v}}(z):=\sum_{i} z^{i} v_{i}
$$

This kind of generating function is common enough in probability theory, as it forms the basis of a quite successful probabilistic method [1,2]. A bit more unusual however is the generating function of a matrix $P$, which is defined as

$$
\mathcal{G}_{P}(x, y):=\sum_{i} \sum_{j} x^{i} p_{i j} y^{j} .
$$

where we use the convention, here and in the rest of the paper, that sums run over the state space $\mathcal{X}$, unless specified otherwise.

Note: Although all countably infinite sets are isomorphic to $\mathbb{N}$, and hence the above definitions should be sufficient, it is sometimes unnatural to invoke 
such isomorphism. For example, when considering a random walk on the quarter plane, it is unnatural to consider anything else than bivariate generating functions, of the type $\mathcal{G}_{\mathbf{v}}\left(z_{1}, z_{2}\right):=\sum_{i \in \mathcal{X}} \sum_{j \in \mathcal{X}} z_{1}^{i} z_{2}^{j} v_{i j}$. For the transform of the corresponding transition matrix, the same line of reasoning results in a fourdimensional generating function.

We shall now be concerned with finding the fundamental kernel $Z$, which has the property that if a solution to Poisson's equation exists, then $\mathbf{h}=Z \mathbf{f}$ is the solution for which $\boldsymbol{\pi h}=\boldsymbol{\pi} \mathbf{f} .{ }^{1}$ The matrix $Z$ satisfies (see e.g. [6]):

$$
Z=(I-P+\mathbf{1} \pi)^{-1},
$$

whenever the inverse exists as a bounded linear operator.

The main starting point is the equation

$$
Z(I-P+\mathbf{1} \pi)=I,
$$

which in the transform domain translates to

$$
\mathcal{G}_{Z}(x, y)-\mathcal{G}_{Z P}(x, y)+\mathcal{G}_{Z}(x, 1) \mathcal{G}_{\boldsymbol{\pi}}(y)=\frac{1}{1-x y} .
$$

Note that $\mathcal{G}_{Z}(x, 1)=1 /(1-x)$, as $Z \mathbf{1}=\mathbf{1}$, hence we have:

$$
\mathcal{G}_{Z}(x, y)-\mathcal{G}_{Z P}(x, y)=\frac{1}{1-x y}-\frac{\mathcal{G}_{\boldsymbol{\pi}}(y)}{1-x},
$$

which is altogether not too different from the transform version of the invariance equation:

$$
\mathcal{G}_{\boldsymbol{\pi}}(z)-\mathcal{G}_{\boldsymbol{\pi} P}(z)=0
$$

Indeed, in both cases, success of the transform-domain recipe largely depends on whether the expression for $\mathcal{G}_{\boldsymbol{\pi} P}(z)$ (resp. $\mathcal{G}_{Z P}(x, y)$ ) can be conveniently rewritten in terms of $\mathcal{G}_{\boldsymbol{\pi}}(z)$ (resp. $\mathcal{G}_{Z}(x, y)$ ). The examples below seem to indicate that if the transform-domain solution can be found for the invariance equation, the corresponding PE derivation is a bit more tedious, but not by much. In the following subsections, we shall explicitly derive expressions for $\mathcal{G}_{Z}(x, y)$ for a variety of models.

\subsection{Reflected random walks, skip-free to the left}

Let us consider Markov chains with the following often-encountered transition matrix:

$$
P=\left(\begin{array}{cccccc}
b_{0} & b_{1} & b_{2} & b_{3} & \cdots & \\
a_{0} & a_{1} & a_{2} & a_{3} & \cdots & \\
& a_{0} & a_{1} & a_{2} & a_{3} & \cdots \\
& \ddots & \ddots & \ddots & \ddots
\end{array}\right) .
$$

\footnotetext{
${ }^{1}$ It is easily checked that if $\mathbf{h}$ is a solution, then also $\tilde{\mathbf{h}}=\mathbf{h}+c \mathbf{1}$, for any $c$. In this respect, a popular alternative ( [8]) for $Z$ is the deviation matrix $D=Z-\mathbf{1} \boldsymbol{\pi}$, so that $\tilde{\mathbf{h}}=D \mathbf{f}$ is the PE solution for which $\boldsymbol{\pi} \tilde{\mathbf{h}}=\mathbf{0}$.
} 
This Markov chain, which is a random walk on $\mathbb{N}$, reflected at 0 , has found important applications in queueing theory. Firstly, it appears in the study of the continuous-time $M / G / 1$ queue at embedded points, but also in discrete-time queues, it plays an important role. The qualification skip-free to the left refers to the fact that while jumps to the right may be arbitrarily large, jumps to the left are at most of size 1 .

The invariance equation for this Markov chain has been derived many times. We rederive it in the notation of the paper in order to be able to show the similarities and differences with the PE. Let $\mathcal{G}_{\mathbf{a}}(z)=\sum_{i} z^{i} a_{i}, \mathcal{G}_{\mathbf{b}}(z)=\sum_{i} z^{i} b_{i}$ and let $z^{\mathcal{X}}$ stand for the row-vector $\left(1, z, z^{2}, \ldots\right)$. The invariance equation in the transform domain (12), simplifies in this case to:

$$
\begin{aligned}
\mathcal{G}_{\boldsymbol{\pi}}(z) & =\mathcal{G}_{\boldsymbol{\pi} P}(z)=\boldsymbol{\pi} P\left(z^{\mathcal{X}}\right)^{T} \\
& =\boldsymbol{\pi}\left(\mathcal{G}_{\mathbf{b}}(z), \mathcal{G}_{\mathbf{a}}(z), z \mathcal{G}_{\mathbf{a}}(z), z^{2} \mathcal{G}_{\mathbf{a}}(z), \ldots\right)^{T} \\
& =\pi_{0} \mathcal{G}_{\mathbf{b}}(z)+\sum_{j>0} \pi_{j} z^{j-1} \mathcal{G}_{\mathbf{a}}(z) \\
& =\mathcal{G}_{\boldsymbol{\pi}}(0) \mathcal{G}_{\mathbf{b}}(z)+\left(\mathcal{G}_{\boldsymbol{\pi}}(z)-\mathcal{G}_{\boldsymbol{\pi}}(0)\right) \frac{\mathcal{G}_{\mathbf{a}}(z)}{z} .
\end{aligned}
$$

The only unknown in this equation is $\mathcal{G}_{\boldsymbol{\pi}}(0)$, which we can find by differentiating the above equation with respect to $z$ and substituting $z=1$, which gives after some manipulations:

$$
\mathcal{G}_{\boldsymbol{\pi}}(0)=\frac{1-\mathcal{G}_{\mathbf{a}}^{\prime}(1)}{1-\mathcal{G}_{\mathbf{a}}^{\prime}(1)+\mathcal{G}_{\mathbf{b}}^{\prime}(1)} .
$$

This leads to the following final expression for $\mathcal{G}_{\boldsymbol{\pi}}(z)$ :

$$
\mathcal{G}_{\boldsymbol{\pi}}(z)=\frac{1-\mathcal{G}_{\mathbf{a}}^{\prime}(1)}{1-\mathcal{G}_{\mathbf{a}}^{\prime}(1)+\mathcal{G}_{\mathbf{b}}^{\prime}(1)} \frac{z \mathcal{G}_{\mathbf{b}}(z)-\mathcal{G}_{\mathbf{a}}(z)}{z-\mathcal{G}_{\mathbf{a}}(z)} .
$$

For Poisson's equation, we need an expression for $\mathcal{G}_{Z P}(x, y)$ :

$$
\begin{aligned}
\mathcal{G}_{Z P}(x, y) & =x^{\mathcal{X}} Z P\left(y^{\mathcal{X}}\right)^{T} \\
& =x^{\mathcal{X}} Z\left(\mathcal{G}_{\mathbf{b}}(y), \mathcal{G}_{\mathbf{a}}(y), y \mathcal{G}_{\mathbf{a}}(y), y^{2} \mathcal{G}_{\mathbf{a}}(y), \ldots\right)^{T} \\
& =\left(x^{\mathcal{X}} Z\right)_{0} \mathcal{G}_{\mathbf{b}}(y)+\sum_{j>0}\left(x^{\mathcal{X}} Z\right)_{j} y^{j-1} \mathcal{G}_{\mathbf{a}}(y) \\
& =\mathcal{G}_{Z}(x, 0) \mathcal{G}_{\mathbf{b}}(y)+\left(\mathcal{G}_{Z}(x, y)-\mathcal{G}_{Z}(x, 0)\right) \frac{\mathcal{G}_{\mathbf{a}}(y)}{y} .
\end{aligned}
$$

which leads to

$$
\mathcal{G}_{Z}(x, y)\left[y-\mathcal{G}_{\mathbf{a}}(y)\right]-\mathcal{G}_{Z}(x, 0)\left[y \mathcal{G}_{\mathbf{b}}(y)-\mathcal{G}_{\mathbf{a}}(y)\right]=\frac{y}{1-x y}-\frac{y \mathcal{G}_{\boldsymbol{\pi}}(y)}{1-x} .
$$

Still unknown in this equation is the function $\mathcal{G}_{Z}(x, 0)$, which we can recover with almost exactly the same trick as before: we differentiate to $y$ and then substitute $y=1$. 


$$
\mathcal{G}_{Z}(x, 0)=\frac{1-\mathcal{G}_{\mathbf{a}}^{\prime}(1)+\mathcal{G}_{\pi}^{\prime}(1)-x /(1-x)}{\left(1-\mathcal{G}_{\mathbf{a}}^{\prime}(1)+\mathcal{G}_{\mathbf{b}}^{\prime}(1)\right)(1-x)}
$$

\subsection{Reflected random walks, limited displacement}

We now consider a generalization of the previous section that allows jumps of maximally size $c$ to the left (the standard term limited displacement refers to exactly this restriction in leftward jumps):

$$
P=\left(\begin{array}{cccccc}
b_{0,0} & b_{0,1} & b_{0,2} & b_{0,3} & \cdots & \\
b_{1,0} & b_{1,1} & b_{1,2} & b_{1,3} & \cdots & \\
\vdots & \vdots & \vdots & \vdots & \\
b_{c-1,0} & b_{c-1,1} & b_{c-1,2} & b_{c-1,3} & \cdots & \\
a_{0} & a_{1} & a_{2} & a_{3} & \cdots & \\
& a_{0} & a_{1} & a_{2} & a_{3} & \cdots \\
& & \ddots & \ddots & \ddots
\end{array}\right)
$$

This type of Markov chain finds applications in queues with multiple servers, or in queues with batch servers. Let $\mathcal{G}_{\mathbf{a}}(z)=\sum_{i} z^{i} a_{i}$ and $\mathcal{G}_{\mathbf{b}_{i}}(z)=\sum_{j} z^{j} b_{i, j}$.

We rederive the stationary distribution $\boldsymbol{\pi}$ which leads to

$$
\begin{aligned}
\mathcal{G}_{\boldsymbol{\pi}}(z) & =\boldsymbol{\pi}\left(\mathcal{G}_{\mathbf{b}_{0}}(z), \ldots, \mathcal{G}_{\mathbf{b}_{c-1}}(z), \mathcal{G}_{\mathbf{a}}(z), z \mathcal{G}_{\mathbf{a}}(z), \ldots\right)^{T} \\
& =\sum_{i=0}^{c-1} \pi_{i} \mathcal{G}_{\mathbf{b}_{i}}(z)+\sum_{i=c}^{\infty} \pi_{i} z^{i-c} \mathcal{G}_{\mathbf{a}}(z) \\
& =\sum_{i=0}^{c-1} \pi_{i} \mathcal{G}_{\mathbf{b}_{i}}(z)+\left[\mathcal{G}_{\boldsymbol{\pi}}(z)-\sum_{i=0}^{c-1} \pi_{i} z^{i}\right] \frac{\mathcal{G}_{\mathbf{a}}(z)}{z^{c}} .
\end{aligned}
$$

After some elementary manipulations, this gives rise to

$$
\mathcal{G}_{\boldsymbol{\pi}}(z)\left[z^{c}-\mathcal{G}_{\mathbf{a}}(z)\right]=\sum_{i=0}^{c-1} \pi_{i}\left[z^{c} \mathcal{G}_{\mathbf{b}_{i}}(z)-z^{i} \mathcal{G}_{\mathbf{a}}(z)\right],
$$

where $\pi_{i}$ is the $i$ th component of the stationary vector $\boldsymbol{\pi}$. We can determine the first $c$ components of this vector by computing the zeros inside the unit circle of the equation $\mathcal{G}_{\mathbf{a}}(z)=z^{c}$. Rouché's theorem ensures the existence of exactly $c-1$ zeros $\zeta_{j},\left|\zeta_{j}\right|<1,0<j<c$, in addition to the zero $\zeta_{0}=1$. We thus find a system of linear equations

$$
\sum_{i=0}^{c-1} \pi_{i}\left[\zeta_{j}^{c} \mathcal{G}_{\mathbf{b}_{i}}\left(\zeta_{j}\right)-\zeta_{j}^{i} \mathcal{G}_{\mathbf{a}}\left(\zeta_{j}\right)\right]=0, \text { for } 0<j<c .
$$

For the PE, we likewise get: 


$$
\begin{aligned}
\mathcal{G}_{Z P}(x, y) & =x^{\mathcal{X}} Z\left(\mathcal{G}_{\mathbf{b}_{0}}(y), \ldots, \mathcal{G}_{\mathbf{b}_{c-1}}(y), \mathcal{G}_{\mathbf{a}}(y), y \mathcal{G}_{\mathbf{a}}(y), \ldots\right)^{T} \\
& =\sum_{i=0}^{c-1} Z_{i}(x) \mathcal{G}_{\mathbf{b}_{i}}(y)+\sum_{i=c}^{\infty} Z_{i}(x) y^{i-c} \mathcal{G}_{\mathbf{a}}(y) \\
& =\sum_{i=0}^{c-1} Z_{i}(x) \mathcal{G}_{\mathbf{b}_{i}}(y)+\left[\mathcal{G}_{Z}(x, y)-\sum_{i=0}^{c-1} Z_{i}(x) y^{i}\right] \frac{\mathcal{G}_{\mathbf{a}}(y)}{y^{c}}
\end{aligned}
$$

where $Z_{j}(x)=\sum_{i} x^{i} Z_{i j}$. Substituting this into (11), we obtain:

$$
\mathcal{G}_{Z}(x, y)\left[y^{c}-\mathcal{G}_{\mathbf{a}}(y)\right]-\sum_{i=0}^{c-1} Z_{i}(x)\left[z^{c} \mathcal{G}_{\mathbf{b}_{i}}(z)-z^{i} \mathcal{G}_{\mathbf{a}}(z)\right]=\frac{y^{c}}{1-x y}-\frac{y^{c} \mathcal{G}_{\boldsymbol{\pi}}(y)}{1-x},
$$

As with the invariance equation we equally get a system of linear equations by substituting $y$ by $\zeta_{k}, k=0, \ldots, c-1$. The equation for $k=0$ is replaced by the equation obtained by deriving (25) w.r.t. $y$ and substituting $y$ by 1 , since the former does not provide any additional information.

$$
\sum_{i=0}^{c-1} Z_{j}(x)\left(j-c+\mathcal{G}_{\mathbf{a}}^{\prime}(1)-\mathcal{G}_{\mathbf{b}_{i}}^{\prime}(1)\right)=\frac{x /(1-x)-\mathcal{G}_{\boldsymbol{\pi}}^{\prime}(1)+\mathcal{G}_{\mathbf{a}}^{\prime}(1)-c}{1-x} .
$$

Hence we see that all $Z_{j}(x)$ are linear combinations of the following form:

$$
Z_{j}(x)=\sum_{k=0}^{c-1} \frac{d_{k j}}{1-x \zeta_{k}}+\frac{d_{c j}}{(1-x)^{2}}
$$

so that $Z_{j}(x)$ can have no other poles than $\zeta_{k}^{-1}$, and the problem is reduced to finding the different $d_{k j}$. Introduce matrices $A$ and $B$ where $A$ is $c \times c$ and $B$ is $(c+1) \times c$, and there elements are given as follows:

$$
\begin{gathered}
A_{j k}=j-c+\mathcal{G}_{\mathbf{a}}^{\prime}(1)-\mathcal{G}_{\mathbf{b}_{j}}^{\prime}(1), k=0 \\
=\zeta_{k}^{j}-\mathcal{G}_{\mathbf{b}_{j}}\left(\zeta_{k}\right), k>0 \\
B=\left(\begin{array}{ccccc}
{\left[\mathcal{G}_{\mathbf{a}}^{\prime}(1)-\mathcal{G}_{\boldsymbol{\pi}}^{\prime}(1)-c\right]} & -\mathcal{G}_{\boldsymbol{\pi}}\left(\zeta_{1}\right) & -\mathcal{G}_{\boldsymbol{\pi}}\left(\zeta_{2}\right) & \cdots & -\mathcal{G}_{\boldsymbol{\pi}}\left(\zeta_{c-1}\right) \\
0 & 1 & & \cdots & 0 \\
0 & & 1 & \cdots & 0 \\
\vdots & & & \ddots & \vdots \\
0 & & & \cdots & 1 \\
x & 0 & 0 & \cdots & 0
\end{array}\right)
\end{gathered}
$$


Then the $(i, j)$ 'th element of $B A^{-1}$ is exactly $d_{i, j}$. Note that apart from the first row, $A$ can also be used for the invariance equation above (for obtaining $\pi_{j}$, $j=0, \ldots, c-1)$. Therefore in essence solving Poisson's equation is numerically not harder than solving the invariance equation.

\subsection{Extracting information from $\mathcal{G}_{Z}(x, y)$}

In this section, we explore how to get information on the infinite matrix $Z$ by means of generating function $\mathcal{G}_{Z}(x, y)$. Specifically, we focus on results of the form $\phi Z \mathbf{f}$ for a given row vector $\phi$ and a column vector $\mathbf{f}$. First, note that $Z(x, y)$ is in itself a result of this form, with 'geometric' vectors $\phi=\left[x^{i}\right]_{i \in \mathbb{N}}$ and $\mathbf{f}=\left[y^{i}\right]_{i \in \mathbb{N}}$. We first consider the special case for which $\phi$ is geometric but $\mathbf{f}$ has a more general form, so that $\phi Z \mathbf{f}=\mathcal{G}_{\mathbf{h}}(x)$, the generating function of the value function corresponding with cost function $\mathbf{f}$. We assume that all but finitely many $f_{i}$ have the same sign, so that we can indeed swap sum and integral in the following derivation:

$$
\begin{aligned}
\mathcal{G}_{\mathbf{h}}(x) & =\sum_{i, j} x^{i} Z_{i j} f_{j} \\
& =\frac{1}{2 \pi \imath} \sum_{j} f_{j} \oint \mathcal{G}_{Z}(x, y) y^{-j-1} d y \\
& =\frac{1}{2 \pi \imath} \oint \mathcal{G}_{Z}(x, y) \mathcal{G}_{\mathbf{f}}\left(y^{-1}\right) y^{-1} d y
\end{aligned}
$$

where the integrals are taken along a suitable contour. Evaluation can proceed numerically by approximating the integral by a sum, which in fact amounts to the application of fast Fourier transform techniques (FFT). Such approximation techniques are not required if $\mathbf{f}$ has one of the two fundamental forms:

$$
f_{j}^{(1)}=j^{n} \alpha^{j} \quad \text { or } \quad f_{j}^{(2)}=\delta_{j}^{k} .
$$

We have that $\mathcal{G}_{\mathbf{h}^{(1)}}(x)=\mathcal{G}_{Z \mathbf{f}^{(1)}}(x)$ reduces to

$$
\mathcal{G}_{\mathbf{h}^{(1)}}(x)=\left.\left(y \frac{\partial}{\partial y}\right)^{n} \mathcal{G}_{Z}(x, y)\right|_{y=\alpha},
$$

while $\mathcal{G}_{\mathbf{h}^{(2)}}(x)=\mathcal{G}_{Z_{\mathbf{f}^{(2)}}}(x)$ can be written as:

$$
\mathcal{G}_{\mathbf{h}^{(2)}}(x)=\left.\frac{\partial^{k}}{\partial y^{k}} \mathcal{G}_{Z}(x, y)\right|_{y=0}
$$

Note that we have closed-form expressions for the generating function of the value function if $\mathbf{f}$ is a (finite) linear combination of such forms as well. We denote the set of such functions as $C$, which is closed under both addition and multiplication. It contains all constant, polynomial, exponential, functions and all linear combinations and products of such functions. As such, it is a dense set 
on the set of functions $f: \mathcal{X} \rightarrow \mathbb{R}$, meaning that any function can be arbitrarily closely approximated.

We now derive integral expressions for $\phi Z \mathbf{f}$ :

$$
\begin{aligned}
\phi Z \mathbf{f} & =\sum_{i, j} \phi_{i} Z_{i j} f_{j} \\
& =-\sum_{i j} \phi_{i} f_{j} \frac{1}{4 \pi^{2}} \oint \oint d x d y x^{-i-1} \mathcal{G}_{Z}(x, y) y^{-j-1} \\
& =-\frac{1}{4 \pi^{2}} \oint \oint d x d y \mathcal{G}_{\phi}\left(x^{-1}\right) x^{-1} \mathcal{G}_{Z}(x, y) \mathcal{G}_{\mathbf{f}}\left(y^{-1}\right) y^{-1}
\end{aligned}
$$

In case $\phi$ is also of a fundamental form:

$$
\phi_{j}^{(1)}=j^{m} \beta^{j} \quad \text { or } \quad \phi_{j}^{(2)}=\delta_{j}^{\ell},
$$

we obtain for each of the four options a different expression for $\phi Z \mathbf{f}$ :

$$
\begin{aligned}
\phi^{(1)} Z \mathbf{f}^{(1)} & =\left.\left(x \frac{\partial}{\partial x}\right)^{m}\left(y \frac{\partial}{\partial y}\right)^{n} \mathcal{G}_{Z}(x, y)\right|_{(x, y)=(\beta, \alpha)}, \\
\phi^{(1)} Z \mathbf{f}^{(2)} & =\left.\left(x \frac{\partial}{\partial x}\right)^{m} \frac{1}{k !} \frac{\partial^{k}}{\partial y^{k}} \mathcal{G}_{Z}(x, y)\right|_{(x, y)=(\beta, 0)}, \\
\phi^{(2)} Z \mathbf{f}^{(1)} & =\left.\frac{1}{\ell !} \frac{\partial^{\ell}}{\partial x^{\ell}}\left(y \frac{\partial}{\partial y}\right)^{n} \mathcal{G}_{Z}(x, y)\right|_{(x, y)=(0, \alpha)}, \\
\phi^{(2)} Z \mathbf{f}^{(2)} & =\left.\frac{1}{\ell !} \frac{\partial^{\ell}}{\partial x^{\ell}} \frac{1}{k !} \frac{\partial^{k}}{\partial y^{k}} \mathcal{G}_{Z}(x, y)\right|_{(x, y)=(0,0)}
\end{aligned}
$$

\section{Application to asymptotic variance}

The asymptotic variance (see e.g. [6]) of a functional $f$ on a Markov chain $\left\{X_{n}\right\}$ is defined as follows:

$$
\gamma_{f}^{2}=\lim _{N \rightarrow \infty} \frac{1}{N} \mathbb{E}:\left[\sum_{n=0}^{N-1}\left(f\left(X_{n}\right)-\bar{f}\right)^{2}\right],
$$

where $\bar{f}=\boldsymbol{\pi} \mathbf{f}$, i.e. the expectation of $f$ under stationarity.

This concept plays a key role in establishing a central limit theorem (CLT) for Markov chains, indeed, under some fairly broad conditions [6], we have that the sequence

$$
\left(N \gamma_{f}^{2}\right)^{-1 / 2} \sum_{n=0}^{N-1}\left(f\left(X_{n}\right)-\bar{f}\right),
$$

converges to a normal distribution with zero mean and unit variance as $N \rightarrow \infty$.

This property has many important applications, for example in planning simulations, but also in heavy-traffic theory, which we will illustrate in Sec. 3.2. 


\subsection{Calculation of $\gamma_{f}^{2}$}

Calculating $\gamma_{f}^{2}$ directly from the formula (40) often leads to long-winded derivations, which can be alleviated if we carry it out in the following way: for $\mathbf{h}=Z \mathbf{f}$, we have (see [6]):

$$
\begin{aligned}
\gamma_{f}^{2} & =\sum_{i} \pi_{i}\left(\left(h_{i}\right)^{2}-\left(h_{i}-f_{i}+\bar{f}\right)^{2}\right) \\
& =2 \sum_{i} \pi_{i} h_{i} f_{i}-\sum_{i} \pi_{i}\left(f_{i}\right)^{2}-(\bar{f})^{2} .
\end{aligned}
$$

where we made use of the fact that $\boldsymbol{\pi} \mathbf{h}=\boldsymbol{\pi} \mathbf{f}$. Note that only the first term involves $\mathbf{h}$. We show how to compute this term by using the techniques of Sec. 2.3. Note that

$$
\sum_{i} \pi_{i} h_{i} f_{i}=\sum_{i, j} \pi_{i} f_{i} Z_{i j} f_{j}
$$

If both $\pi_{i}$ and $f_{i}$ belong to the set $C$ as defined in Sec. 2.3, then we obtain a closed-form expression for the asymptotic variance. This implies that $\mathcal{G}_{\boldsymbol{\pi}}(z)$ must be a rational function.

\subsection{An application to heavy traffic}

Since Kingman [16], heavy-traffic theory is an important topic in queueing theory. In the following, we give a very short account of the results for a single queue (i.e. the $G / G / 1$ model). In keeping with the conventions of rest of the paper, we stick to a discrete-time scenario, although extensions to continuous time are fairly straight-forward. Consider a queue with a stationary but not necessarily independent input process $\left\{A_{n}\right\}$ and a capacity $c$. The virtual work $W_{n}$ at time instant $n$ is given by the recursive equation

$$
W_{n+1}=\left(W_{n}+A_{n}-c\right)^{+}
$$

Heavy-traffic theory is concerned with the limit as $\mathbb{E}:\left[A_{0}\right] \uparrow c$ and more specifically states that if the process $\left\{A_{n}\right\}$ admits a CLT with mean $\rho$ and asymptotic variance $\gamma^{2}$, then the mean virtual work in stationarity is equal to

$$
E\left[W_{\infty}\right]=\frac{\gamma^{2}}{2(c-\rho)}
$$

Moreover the stationary distribution converges to an exponential distribution with a mean given by the above display.

In order to apply the results of this paper to a concrete queueing scenario. We consider a two-queue model where the content of the first queue serves as the input for the second queue:

$$
\left(U_{n+1}^{(1)}, U_{n+1}^{(2)}\right)=\left(\left[U_{n}^{(1)}+A_{n}-1\right]^{+},\left[U_{n}^{(2)}+U_{n}^{(1)}-1\right]^{+}\right)
$$


This may seem like an artificial model, but it might be used in the following scenario. People are waiting in line for a service (for example, buying a ticket at the movies), and while doing so, they make use of an internet hotspot, thus creating internet traffic proportional to the number of waiting customers. A more pragmatic reason is that it allows us to make direct use of the results for the class of Markov chains of Sec. 2.1. With some extra work, e.g. by applying our recipe to the two-dimensional Markov chain $\left\{\left(U_{n}^{(1)}, D_{n}^{(1)}\right)\right\}$, where $D_{n}^{(1)}$ denotes the number of departures from the first queue, we may also consider more traditional scenarios such as tandem queues etc., which in fact leads to the asymptotic variance of departure processes as considered eg. in [15].

If we assume the second queue to be operating close to its maximal capacity, then we can apply the aforementioned heavy-traffic results, as the sequence $\left\{U_{n}^{(1)}\right\}$ forms a (dependent) input process for the second queue. We compute the value of $\gamma_{f}^{2}$ in closed form. Note that in this particular case, $f_{i}=i$. Let us apply the results of Sec. 2.1 with $\mathcal{G}_{\mathbf{a}}(z)=\mathcal{G}_{\mathbf{b}}(z)=a_{0}+a_{1} z+a_{2} z^{2}$. Note that we can derive closed-form solutions under broader conditions that we do here, but we opt for a simple, tractable example. Let $\rho_{1}=a_{1}+2 a_{2}$. We have that $\mathcal{G}_{\pi}(z)=\left(a_{0}-a_{2}\right) /\left(a_{0}-a_{2} z\right)$, such that $\pi_{i}=(1-r) r^{i}$, with $r=a_{2} / a_{0}$. After some (fairly straightforward) algebraic manipulations, we find that

$$
\begin{aligned}
\mathcal{G}_{Z}(x, y)=\frac{1}{a_{0}-a_{2} y}\left(\frac{y\left(a_{0} x-a_{2}\right)}{(1-x)\left(a_{0}-a_{2} y\right)(1-x y)}\right. & \\
& \left.+\frac{a_{0}-a_{2}+a_{0} /\left(a_{0}-a_{2}\right)}{1-x}-\frac{x}{(1-x)^{2}}\right) .
\end{aligned}
$$

Eq. (41) specializes in this case to

$$
\gamma^{2}=2(1-r) \phi Z \mathbf{f}-\frac{a_{0} a_{2}+2 a_{2}^{2}}{\left(a_{0}-a_{2}\right)^{2}} .
$$

Note that $\phi_{i}=i r^{i}$ and $f_{i}=i$, so that

$$
\phi Z \mathbf{f}=\left.\frac{\partial^{2}}{\partial x \partial y} \mathcal{G}_{Z}(x, y)\right|_{(x, y)=(r, 1)},
$$

which leads to a long but closed-form expression for $\gamma^{2}$.

In Figure 1, we illustrate the results of this section with a numerical example. Note that there are only two degrees of freedom for the the parameters of the first queue. When we fix the load $\rho_{2}$ as seen by the second queue (which is equal to $a_{2} /\left(a_{0}-a_{2}\right)$, the mean content of the first queue), then a plausible choice for the other parameter is given by $a_{1}$, which is a measure for how quickly the queue content process varies. We see that $\gamma^{2}$ increases as $a_{1}$ gets larger.

\section{Further applications and concluding remarks}

As there are plenty of applications for Poisson's equation next to asymptotic variance, we presume that the methods of this paper may be useful in other 


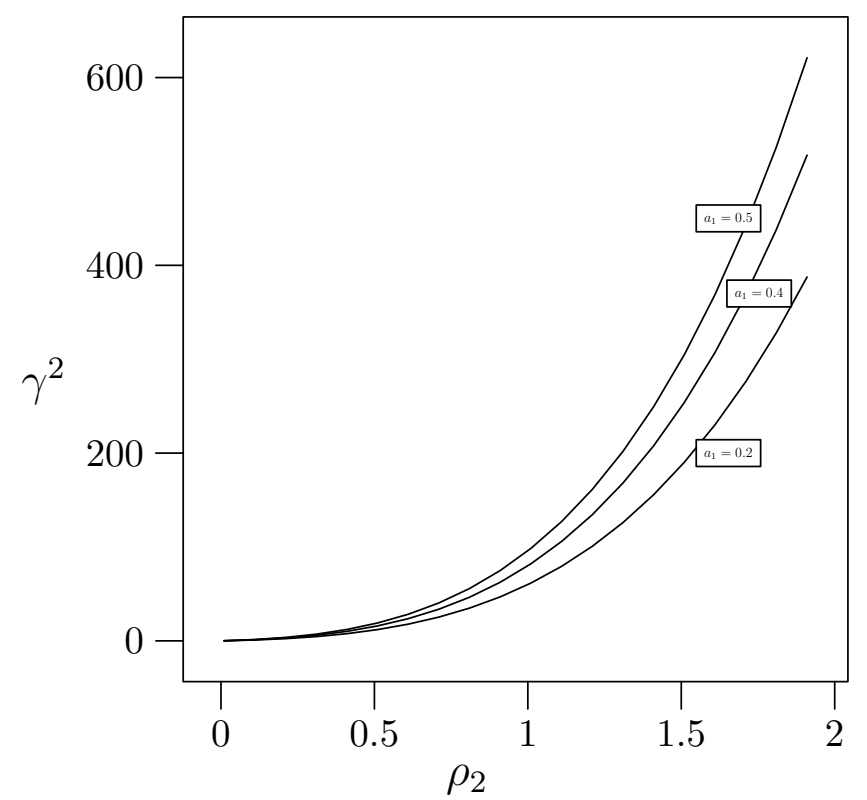

Fig. 1. Plot of the asymptotic variance $\gamma^{2}$ of the input stream against load of the second queue for different values of $a_{1}$.

areas than in asymptotic variance. We give some initial findings on a few of them.

Controlled Markov chains, or Markov decision processes, are perhaps the most obvious application. As this work may help find efficient solution methods for the value function (often a rather costly step), it may lead to better algorithms for some classes of MDP. It seems of utmost importance however that every policy leads to a Markov chain that is sufficiently structured.

Another application is perturbation of Markov chains. Consider a family of Markov chains depending on a parameter $\alpha$ with transition matrices $P^{(\alpha)}$. For small values of $\alpha$, The central formula for the stationary vector of a perturbed system is as follows:

$$
\boldsymbol{\pi}^{(\alpha)}=\boldsymbol{\pi}^{(0)} \sum_{k}\left(\left(P^{(\alpha)}-P^{(0)}\right)\left(Z^{(0)}-\mathbf{1} \boldsymbol{\pi}^{(0)}\right)\right)^{k} .
$$

Although perturbation problems can sometimes be tackled in the transform domain without a detour to PE (see eg. [19]), the approach of this paper may lead to a higher genericity, and potentially to more insight.

Summing up, we have derived transform-based solutions of Poisson's equation for some frequently encountered types of Markov chains and have pointed out some applications. We show that the transform domain may form an attractive tool for researchers working with Poisson's equation, and also that Poisson's 
equation may offer new results and applications for models that allow transformbased solutions.

\section{References}

1. J. Cohen, The single-server queue. North-Holland Series in Appl Math. and Mech., 1969.

2. H. Takagi, Queueing analysis, a foundation of performance evaluation, Vol. 3: Discrete-time systems, Elsevier Science Publishers BV, Amsterdam, The Netherlands, 1993.

3. R. Bellman, On the Theory of Dynamic Programming, Proceedings of the National Academy of Sciences, 1952.

4. Peter W. Glynn and Sean P. Meyn, A Liapounov bound for solutions of the Poisson equation. Ann. Probab. Volume 24, Number 2 (1996), 916-931.

5. Schweitzer. Perturbation theory and finite Markov chains. J. Appl. Prob. 5:401403, 1968.

6. S.P. Meyn, R.L. Tweedie. Markov chains and stochastic stability. Springer-Verlag, London, 2003

7. S. P. Meyn. Control Techniques for Complex Networks. Cambridge University Press, 2007.

8. S. Asmussen. Applied probability and queues, 2nd edition. Springer, 2003.

9. Neveu, J. Potentiel Markovien récurrent des chaînes de Harris. Ann. Inst. Fourier (Grenoble) 227130 (1972).

10. G.L. Jones. On the Markov chain central limit theorem. Probab, Surv. 1:299-320, 2004.

11. Nummelin, E. On the Poisson equation in the potential theory of a single kernel. Math. Scand. 685982 (1991)

12. Shwartz, A. and Makowski, A. On the Poisson equation for Markov chains: existence of solutions and parameter dependence. Technical report, Dept. Electrical Engineering, Technion - Israel Institute of Technology (1991).

13. G. Koole, F. Spieksma, On deviation matrices for birth-death processes. Probability in the Engineering and Informational Sciences 15:239-258, 2001.

14. A. Hordijk and F.M. Spieksma. On ergodicity and recurrence properties of a Markov chain with an application. Adv. Appl. Probab., 24:343-376, 1992.

15. Nazarathy Y., The Variance of Departure Processes: Puzzling Behavior and Open Problems, Queueing Systems, 68, pp 385-394, (2011).

16. J. F. C. Kingman, On Queues in Heavy Traffic. Journal of the Royal Statistical Society. Series B (Methodological), Vol. 24, No. 2 (1962), pp. 383-392

17. L.B. White. A new policy evaluation algorithm for Markov decision processes with quasi birth-death structure. Stochastic Models, 21:785797, 2005.

18. J. Lambert, B. van Houdt, C. Blondia, A policy iteration algorithm for Markov decision processes skip-free in one direction. Numerical Methods for Structured Markov Chains, 2007.

19. D. Fiems, B. Prabhu, K. De Turck, Analytic approximations of queues with lightlyand heavily-correlated autoregressive service times, Annals of Operations research. 